\title{
Generalized Descents and Normality
}

\author{
Miklós Bóna \\ Department of Mathematics \\ University of Florida \\ Gainesville FL 32611-8105
}

Submitted: Jan 29, 2008; Accepted: Jun 11, 2008; Published: Jun 20, 2008

Mathematics Subject Classification: 05A16

\begin{abstract}
We use Janson's dependency criterion to prove that the distribution of $d$-descents of permutations of length $n$ converge to a normal distribution as $n$ goes to infinity. We show that this remains true even if $d$ is allowed to grow with $n$.
\end{abstract}

\section{Introduction}

Let $p=p_{1} p_{2} \cdots p_{n}$ be a permutation. We say that the pair $(i, j)$ is a $d$-descent in $p$ if $i<$ $j \leq i+d$, and $p_{i}>p_{j}$. In particular, 1-descents correspond to descents in the traditional sense, and $(n-1)$-descents correspond to inversions. This concept was introduced in [2] by De Mari and Shayman, whose motivation came from algebraic geometry. They have proved that if $n$ and $d$ are fixed, and $c_{k}$ denotes the number of permutations of length $n$ with exactly $k d$-descents, then the sequence $c_{0}, c_{1}, \cdots$ is unimodal, that is, it increases steadily, then it decreases steadily. It is not known in general if the sequence $c_{0}, c_{1}, \cdots$ is log-concave or not, that is, whether $c_{k-1} c_{k+1} \leq c_{k}^{2}$ holds for all $k$. We point out that in general, the polynomial $\sum_{k} c_{k} x^{k}$ does not have real roots only. Indeed, in the special case of $d=n-1$, we get the well-known [1] identity

$$
\sum_{k} c_{k} x^{k}=(1+x) \cdot\left(1+x+x^{2}\right) \cdots\left(1+x+\cdots+x^{n-1}\right)
$$

which has all $n$th roots of unity as roots. Indeed, in this case, a $d$-descent is just an inversion, as we said above.

In this paper, we prove a related property of generalized descents by showing that their distribution converges to a normal distribution as the length $n$ of our permutations goes to infinity. Our main tool is Janson's dependency criterion, which is a tool to prove normality for sums of bounded random variables with a sparse dependency graph. While the proof itself is reasonably straightforward, we find the very fact that Janson's criterion 
is being applied to objects usually studied by algebraic, not probabilistic combinatorialists, interesting. For results of similar flavor, the reader is encouraged to consult Jason Fulman's papers [5] and [6].

\section{The Proof of Asymptotic Normality}

\subsection{Background and Definitions}

We need to introduce some notation for transforms of the random variable $Z$. Let $\bar{Z}=$ $Z-E(Z)$, let $\tilde{Z}=\bar{Z} / \sqrt{\operatorname{Var}(Z)}$, and let $Z_{n} \rightarrow N(0,1)$ mean that $Z_{n}$ converges in distribution to the standard normal variable.

For the rest of this section, let $d \geq 1$ be a fixed positive integer. Let $X_{n}=X_{n}^{(d)}$ denote the random variable counting the $d$-descents of a randomly selected permutation of length $n$. We want to prove that $X_{n}$ converges to a normal distribution as $n$ goes to infinity, in other words, that $\tilde{X}_{n} \rightarrow N(0,1)$ as $n \rightarrow \infty$. Our main tool in doing so is a theorem called Janson's dependency criterion. In order to state that theorem, we need the following definition.

Definition 1 Let $\left\{Y_{n, k} \mid k=1,2 \cdots\right\}$ be an array of random variables. We say that a graph $G$ is a dependency graph for $\left\{Y_{n, k} \mid k=1,2 \cdots\right\}$ if the following two conditions are satisfied:

1. There exists a bijection between the random variables $Y_{n, k}$ and the vertices of $G$, and

2. If $V_{1}$ and $V_{2}$ are two disjoint sets of vertices of $G$ so that no edge of $G$ has one endpoint in $V_{1}$ and another one in $V_{2}$, then the corresponding sets of random variables are independent.

Note that the dependency graph of a family of variables is not unique. Indeed if $G$ is a dependency graph for a family and $G$ is not a complete graph, then we can get other dependency graphs for the family by simply adding new edges to $G$.

Now we are in position to state Janson's dependency criterion.

Theorem 1 [7] Let $Y_{n, k}$ be an array of random variables such that for all $n$, and for all $k=1,2, \cdots, N_{n}$, the inequality $\left|Y_{n, k}\right| \leq A_{n}$ holds for some real number $A_{n}$, and that the maximum degree of a dependency graph of $\left\{Y_{n, k} \mid k=1,2, \cdots, N_{n}\right\}$ is $\Delta_{n}$.

Set $Y_{n}=\sum_{k=1}^{N_{n}} Y_{n, k}$ and $\sigma_{n}^{2}=\operatorname{Var}\left(Y_{n}\right)$. If there is a natural number $m$ so that

$$
N_{n} \Delta_{n}^{m-1}\left(\frac{A_{n}}{\sigma_{n}}\right)^{m} \rightarrow 0
$$

then

$$
\tilde{Y}_{n} \rightarrow N(0,1)
$$




\subsection{Applying Janson's Criterion}

Recall that in this section, $d$ is a fixed positive integer. We are going to prove that the distribution of $d$-descents of permutations of length $n$ converges to a normal distribution as $n$ goes to infinity.

We will apply Janson's theorem with the $Y_{n, k}$ being the indicator random variables $X_{n, k}$ of the event that a given ordered pair of indices (indexed by $k$ in some way) forms a $d$-descent in the randomly selected permutation $p=p_{1} p_{2} \cdots p_{n}$. So $N_{n}$ is the number of pairs $(i, j)$ of indices so that $1 \leq i<j \leq i+d \leq n$. Then by definition,

$$
Y_{n}=\sum_{k=1}^{N_{n}} Y_{n, k}=\sum_{k=1}^{N_{n}} X_{n, k}=X_{n}
$$

There remains the task of verifying that the variables $Y_{n, k}$ satisfy all conditions of Theorem 1.

First, it is clear that $N_{n} \leq n d$, and we will compute the exact value of $N_{n}$ later. By the definition of indicator random variables, we have $\left|Y_{n, k}\right| \leq 1$, so we can set $A_{n}=1$ for all $n$.

Next we consider the numbers $\Delta_{n}$ in the following dependency graph of the family of the $Y_{n, k}$. Clearly, the indicator random variables that belong to two pairs $(i, j)$ and $(r, s)$ of indices are independent if and only if the sets $\{i, j\}$ and $\{r, s\}$ are disjoint. So fixing $(i, j)$, we need one of $i=r, i=s, j=r$ or $j=s$ to be true for the two distinct variables to be dependent. So let the vertices of $G$ be the $N_{n}$ pairs of indices $(i, j)$ so that $i<j \leq i+d$, and connect $(i, j)$ to $(r, s)$ if one of $i=r, i=s, j=r$ or $j=s$ holds. The graph defined in this way is a dependency graph for the family of the $Y_{n, k}$. Indeed, if $V_{1}$ and $V_{2}$ are two disjoint sets of vertices of this graph, and there is no edge connecting a vertex in $V_{1}$ to a vertex in $V_{2}$, then there is no index $i$ that is present in at least one pair of indices belonging to $V_{1}$ and at least one pair of indices belonging $V_{2}$. So the set of indices present in pairs corresponding to vertices in $V_{1}$ and the set of indices present in pairs corresponding to vertices in $V_{2}$ are disjoint, and therefore, set of variables corresponding to $V_{1}$ and the set of variables corresponding to $V_{2}$ are independent.

For a fixed pair $(i, j)$, each of the four equalities $i=r, i=s, j=r$ or $j=s$ occurs at most $d$ times. (For instance, if $i=s$, then $r$ has to be one of $i-1, i-2, \cdots, i-d$.) Therefore, $\Delta_{n} \leq 4 d$.

If we take a new look at (1), we see that the Janson criterion will be satisfied if we can show that $\sigma_{n}$ is large. This is the content of the next lemma.

Lemma 1 If $n \geq 2 d$, then

$$
\operatorname{Var}\left(X_{n}\right)=\frac{6 d n+10 d^{3}-3 d^{2}-d}{72} .
$$

In particular, $\operatorname{Var}\left(X_{n}\right)$ is a linear function of $n$.

Note that in particular, for $d=1$, we get the well-known fact [1] that the variance of Eulerian numbers in permutations of length $n$ is $(n+1) / 12$. 
Proof: By linearity of expectation, we have

$$
\begin{aligned}
\operatorname{Var}\left(X_{n}\right) & =E\left(X_{n}^{2}\right)-\left(E\left(X_{n}\right)\right)^{2} \\
& =E\left(\left(\sum_{k=1}^{N_{n}} X_{n, k}\right)^{2}\right)-\left(E\left(\sum_{k=1}^{N_{n}} X_{n, k}\right)\right)^{2} \\
& =E\left(\left(\sum_{k=1}^{N_{n}} X_{n, k}\right)^{2}\right)-\left(\sum_{k=1}^{N_{n}} E\left(X_{n, k}\right)\right)^{2} \\
& =\sum_{k_{1}, k_{2}} E\left(X_{n, k_{1}} X_{n, k_{2}}\right)-\sum_{k_{1}, k_{2}} E\left(X_{n, k_{1}}\right) E\left(X_{n, k_{2}}\right)
\end{aligned}
$$

Clearly, $E\left(X_{n, k}\right)=1 / 2$, so the $N_{n}^{2}$ summands that appear in the last line of the above chain of equations with a negative sign are each equal to $1 / 4$. As far as the $N_{n}^{2}$ summands that appear with a positive sign, most of them are equal to $1 / 4$. More precisely, if $X_{n, k_{1}}$ and $X_{n, k_{2}}$ are independent, then

$$
E\left(X_{n, k_{1}} X_{n, k_{2}}\right)=E\left(X_{n, k_{1}}\right) E\left(X_{n, k_{2}}\right)=\frac{1}{4} .
$$

If $k_{1}=k_{2}$, then $E\left(X_{n, k_{1}} X_{n, k_{2}}\right)=E\left(X_{k_{1}}^{2}\right)=E\left(X_{k_{1}}\right)=1 / 2$. Otherwise, if $X_{n, k_{1}}$ and $X_{n, k_{2}}$ are dependent, then either $E\left(X_{n, k_{1}} X_{n, k_{2}}\right)=1 / 3$, or $E\left(X_{n, k_{1}} X_{n, k_{2}}\right)=1 / 6$. Indeed, if $X_{k_{1}}$ is the indicator variable of the pair $(i, j)$ being a $d$-descent and $X_{k_{2}}$ is the indicator variable of the pair $(r, s)$ being a $d$-descent, then as we said above, $X_{n, k_{1}}$ and $X_{n, k_{2}}$ are dependent if and only if one of $i=r, i=s, j=r$ or $j=s$ holds. If $i=r$ or $j=s$ holds, then $E\left(X_{n, k_{1}} X_{n, k_{2}}\right)=1 / 3$, and if $i=s$ or $j=r$ holds, then $E\left(X_{n, k_{1}} X_{n, k_{2}}\right)=1 / 6$. Indeed, for instance, with $i=r$, we have $X_{n, k_{1}}=X_{n, k_{2}}=1$ if and only if $p_{i}$ is the largest of the entries $p_{i}, p_{j}$, and $p_{s}$. Similarly, with $i=s$, we have $X_{n, k_{1}}=X_{n, k_{2}}=1$ if and only if $p_{r}>p_{i}>p_{j}$.

We will now count how many summands $E\left(X_{n, k_{1}} X_{n, k_{2}}\right)$ are equal to $1 / 2$, to $1 / 3$, and to $1 / 6$.

1. First, $E\left(X_{n, k_{1}} X_{n, k_{2}}\right)=1 / 2$ if and only if $k_{1}=k_{2}$. This happens $N_{n}$ times, once for each pair $(i, j)$ so that $i<j \leq i+d$. For a given $i$, there are $d$ such pairs if $i \leq n-d$, and $d-t$ such pairs if $i=n-d+t$, so

$$
N_{n}=(n-d) d+(d-1)+(d-2)+\cdots+1=(n-d) d+\left(\begin{array}{l}
d \\
2
\end{array}\right) .
$$

2. Second, $E\left(X_{n, k_{1}} X_{n, k_{2}}\right)=1 / 3$ if $i=r$, or $j=s$. By symmetry, we can consider the first case, then multiply by two. If $i \leq n-d$, then we have $d(d-1)$ choices for $j$ and $s$, and if $i=n-d+t$, then we have $(d-t)(d-t-1)$ choices. So the number of pairs $\left(k_{1}, k_{2}\right)$ so that $E\left(X_{n, k_{1}} X_{n, k_{2}}\right)=1 / 3$ is

$$
2(n-d) d(d-1)+2(d-1)(d-2)+2(d-2)(d-3)+\cdots+2 \cdot 2 \cdot 1=
$$




$$
2(n-d) d(d-1)+4\left(\begin{array}{l}
d \\
3
\end{array}\right)
$$

3. Finally, $E\left(X_{n, k_{1}} X_{n, k_{2}}\right)=1 / 6$ if $i=s$, or $j=r$. By symmetry, we can again consider the first case, then multiply by two. If $d \leq i \leq n-d$, then there are $d^{2}$ choices for $(j, r)$. If $i \leq d$, then there are $d$ choices for $j$, and $i-1$ choices for $r$. If $n-d<i$, then there are $n-i$ choices for $j$, and $d$ choices for $r$, assuming that $n \geq 2 d$. So the number of pairs $\left(k_{1}, k_{2}\right)$ so that $E\left(X_{n, k_{1}} X_{n, k_{2}}\right)=1 / 6$ is

$$
2(n-2 d) d^{2}+2(d-1) d+2(d-2) d+\cdots+2 d=2(n-2 d) d^{2}+d^{2}(d-1) .
$$

For all remaining pairs $\left(k_{1}, k_{2}\right)$, the variables $X_{n, k_{1}}$ and $X_{n, k_{2}}$ are independent, and so $E\left(X_{n, k_{1}} X_{n, k_{2}}\right)=1 / 4$.

Comparing our results from cases 1-3 above with (3), and recalling that in all other cases, $E\left(X_{n, k_{1}} X_{n, k_{2}}\right)=1 / 4$, we obtain the formula that was to be proved. $\diamond$

The proof of the main result of this section is now immediate.

Theorem 2 Let $d$ be a fixed positive integer. Let $X_{n}$ be the random variable counting $d$-descents of a randomly selected n-permutation. Then $\tilde{X}_{n} \rightarrow N(0,1)$.

Proof: Use Theorem 1 with $Y_{n}=X_{n}, \Delta_{n}=4 d, N_{n}=(n-d) d+\left(\begin{array}{l}d \\ 2\end{array}\right)$, and $\sigma_{n}=$ $\sqrt{\frac{6 d n+10 d^{3}-3 d^{2}-d}{72}}$. All we need to show is that there exists a positive integer $m$ so that

$$
\left((n-d) d+\left(\begin{array}{l}
d \\
2
\end{array}\right)\right) \cdot(4 d)^{m-1} \cdot\left(\frac{72}{6 d n+10 d^{3}-3 d^{2}-d}\right)^{m / 2} \rightarrow 0,
$$

for which it suffices to find a positive integer $m$ so that

$$
(d n) \cdot(4 d)^{m-1} \cdot\left(\frac{12}{d n}\right)^{m / 2} \rightarrow 0 .
$$

Clearly, any $m \geq 3$ suffices, since for any such $m$, the left-hand side is of the form $C / n^{\alpha}$, for positive constants $C$ and $\alpha$. $\diamond$

\section{When $d$ grows with $n$}

We see from (7) that the statement of Theorem 2 can be strengthened, from a constant $d$ to a $d$ that is a function of $n$. Indeed, (7) is equivalent to saying that

$$
c n\left(\frac{d}{n}\right)^{m / 2} \rightarrow 0 \text {. }
$$

This convergence holds as long as $d \leq n^{1-\epsilon}$ for some fixed positive $\epsilon$, we can choose $m$ so that $(m / 2) \cdot \epsilon>1$, and then condition $(7)$ will be satisfied. So we have proved the following. 
Proposition 1 Let $n \rightarrow \infty$, and let us assume that there exists a positive constant $\epsilon$ so that for $n$ sufficiently large, $d=d(n) \leq n^{1-\epsilon}$. Let $X_{n}$ be defined as before. Then

$$
\tilde{X}_{n} \rightarrow N(0,1) .
$$

Now let $d$ be such that $n^{0.5}<d \leq n / 2$ holds. Then we can revisit Lemma 1 for another application. Note that as $n \geq 2$, formula (2) implies that

$$
\operatorname{Var} X_{n}>\frac{d^{3}}{8}
$$

Using this estimate for $\sigma_{n}=\sqrt{\operatorname{Var}\left(X_{n}\right)}$ in (1), we see that it suffices to show that there exists a natural number $m$ so that

$$
\left(n d+\left(\begin{array}{l}
d \\
2
\end{array}\right)\right) \cdot(4 d)^{m-1}\left(\frac{8}{d^{3 / 2}}\right)^{m}<\frac{2 d^{3} \cdot 32^{m}}{d^{m / 2}} \rightarrow 0 .
$$

This is clearly true, since any $m>6$ will suffice. Therefore, we have improved our result as follows.

Proposition 2 Let $n \rightarrow \infty$, and let us assume that $d \leq n / 2$. Let $X_{n}$ be defined as before. Then

$$
\tilde{X}_{n} \rightarrow N(0,1) .
$$

This leaves the case of $d>n / 2$. In that case, Lemma 1 has to be modified since we cannot enumerate pairs $\left(k_{1}, k_{2}\right)$ such that $E\left(X_{n, k_{1}} X_{n, k_{2}}\right)=1 / 6$ in the same way as we have done in case 3 of the proof of that lemma. Indeed, no matter what $i$ is, it will never happen that both of $i-d$ and $i+d$ are valid indices.

So assume that $d>n / 2$, and let us count all pairs $\left(k_{1}, k_{2}\right)$ such that $E\left(X_{n, k_{1}} X_{n, k_{2}}\right)=$ $1 / 6$. For symmetry reasons, we can count pairs of indices $(i, j)$ and $(r, s)$ such that $i=s$, and then multiply their number by 2 . The are three subcases to consider

(a) If $1 \leq i \leq n-d$, then we have $i-1$ choices for $r$ and $d$ choices for $j$.

(b) If $n-d+1 \leq i \leq d$, then we have $(i-1)$ choices for $r$, and $n-i$ choices for $j$.

(c) If $d+1 \leq i \leq n$, then we have $d$ choices for $r$ and $n-i$ choices for $j$.

This implies that the number of pairs $\left(k_{1}, k_{2}\right)$ so that $E\left(X_{n, k_{1}} X_{n, k_{2}}\right)=1 / 6$ is

$$
\begin{gathered}
2\left(\sum_{i=1}^{n-d}(i-1) d+\sum_{i=n-d+1} d(i-1)(n-i)+\sum_{i=d+1}^{n} d(n-i)\right)= \\
\frac{-n^{3}+3 n^{2}-2 n+2 d^{3}+6 d^{2}+4 d+6 n^{2} d-6 n d^{2}-12 n d}{3} .
\end{gathered}
$$

The other cases of the proof of Lemma 1 are unchanged. So comparing the new, modified Case 3 to Cases 1 and 2 of Lemma 1 leads to the following lemma. 
Lemma 2 Let $n / 2<d \leq n-1$. Then

$$
\operatorname{Var}\left(X_{n}\right)=\frac{2 n^{3}-6 n^{2}+4 n-12 d^{3}-21 d^{2}-9 d-12 n^{2} d+24 n d^{2}+30 n d+18}{72} .
$$

In particular, we claim that this implies that there exists a positive constant $c$ so that $\operatorname{Var}\left(X_{n}\right)>c n^{3}$ for $n$ sufficiently large. Indeed, let $d=a n$, where $0.5 \leq a \leq 1$. Then the terms of degree three of (9) are

$$
2 n^{3}-12 d^{3}-12 n^{2} d+24 n d^{2}=n^{3}\left(2-12\left(a(a-1)^{2}\right)\right) .
$$

Set $f(a)=12\left(a(a-1)^{2}\right)$, and note that $f^{\prime}(a)=36 a^{2}-48 a+12$ is negative in $a \in[0.5,1)$. So on that interval, $f$ is decreasing, and so its maximal value is $f(0.5)=1.5$. Therefore, the last displayed equation implies that

$$
2 n^{3}-12 d^{3}-12 n^{2} d+24 n d^{2}=n^{3}(2-f(a)) \geq 0.5 n^{3} .
$$

As all other terms on the right-hand side of (9) are of smaller degree, the claim that $\operatorname{Var}\left(X_{n}\right)>c n^{3}$ is proved.

We can now state our comprehensive result.

Theorem 3 Let $n$ and $d$ be positive integers so that $d \leq n$ holds. Let $X_{n}$ count the $d$-descents of a randomly selected permutation of length $n$. Then

$$
\tilde{X}_{n} \rightarrow N(0,1)
$$

Proof: We have previously handled the cases of $d \leq n / 2$, so now we only have to prove the statement for $n / 2<d \leq n$. Apply the Janson Dependency Criterion (Theorem 1) with the estimates $\sigma_{n} \geq c n^{3 / 2}, \Delta_{n} \leq 4 n, A_{n}=1$, and $N_{n} \leq 2 n^{2}$. Then the criterion will be satisfied if we find a natural number $m$ so that

$$
\frac{2 n^{2} \cdot(4 n)^{m-1}}{n^{1.5 m}} \rightarrow 0
$$

as $n$ goes to infinity. Clearly, any $m \geq 3$ will suffice. $\diamond$

\section{Further Directions}

A possible direction for generalizations, suggested by Richard Stanley, is the following. Let $\mathbf{d}=\left(d_{1}, d_{2} \cdots, d_{n-1}\right)$, where the $d_{i}$ are positive integers. If $p=p_{1} \ldots p_{n}$ is in an $n$-permutation, let $f_{d}(p)$ be the number of pairs $(i, j)$ such that $0<j-i \leq d_{i}$ and $p_{i}>p_{j}$. For instance, if $\mathbf{d}=(1,1, \ldots, 1)$ then $f_{d}(p)$ is the number of descents of $p$. If $\mathbf{d}=(n-1, n-2, \ldots, 1)$ then $f_{d}(p)$ is the number of inversions of $p$. It is known [2], by an argument from algebraic geometry, that if

$$
c_{k}=\left|\left\{p \in S_{n}: f_{d}(p)=k\right\}\right|,
$$


then the sequence $c_{0}, c_{1}, \cdots$ is unimodal. Log-concavity and normality are not known. Note that in this paper, we have treated the special case of $\mathbf{d}=(d, d, \cdots, d)$.

\section{Acknowledgment}

I am thankful to Richard Stanley who introduced me to the topic of generalized descents. I am also indebted to Svante Janson who pointed out how to improve my results in this paper.

\section{References}

[1] M. Bóna, Combinatorics of Permutations, CRC Press - Chapman Hall, 2004.

[2] F. De Mari, M. A. Shayman, Generalized Eulerian numbers and the topology of the Hessenberg variety of a matrix. Acta Appl. Math. 12 (1988), no. 3, 213-235.

[3] P. Diaconis, Group Representations in Probability and Statistics, Institute of Mathematical Statistics Lecture Notes, 11, 1988.

[4] J. Fulman, Stein's Method and Non-reversible Markov Chains. Stein's method: expository lectures and applications, 69-77, IMS Lecture Notes Monogr. Ser., 46, Inst. Math. Statist., Beachwood, OH, 2004.

[5] J. Fulman, A Probabilistic Approach to Conjugacy Classes in the Finite Symplectic and Orthogonal Groups; Journal of Algebra. 234 (2000), 207-224.

[6] J. Fulman, Applications of Symmetric Functions to Cycle and Increasing Subsequence Structure After Shuffles, Journal of Algebraic Combinatorics, 16 (2002), 165-194.

[7] Normal convergence by higher semi-invariants with applications to sums of dependent random variables and random graphs. Ann. Prob. 16 (1988), no. 1, 305-312. 